\title{
QUANTITATIVE IMAGE ANALYSIS OF MACULAR DRUSEN FROM FUNDUS PHOTOGRAPHS AND SCANNING LASER OPHTHALMOSCOPE IMAGES
}

\author{
J. N. P. KIRKPATRICK ${ }^{1}$, T. SPENCER ${ }^{2}$, A. MANIVANNAN ${ }^{2}$, P. F. SHARP SHd $^{2}$ and \\ J. V. FORRESTER ${ }^{1}$ \\ Aberdeen
}

\begin{abstract}
SUMMARY
Drusen are common features in the ageing macula and are associated with exudative age-related macular degeneration (ARMD). They appear as characteristic structures in indirect mode infrared scanning laser ophthalmoscope (SLO) images. Manual counting of drusen area in SLO images has shown no significant difference from that in standard fundus photographs in 6 eyes of 5 patients $(p>0.5)$. Computerised image processing techniques have been applied to digitised colour fundus photographs and indirect mode images to quantify the area of the macula affected by drusen in an automated fashion. Application of these methods may permit objective and repeatable assessment of the natural history of macular drusen. For a specificity of $90 \%$, a sensitivity of $60 \%$ for colour fundus photographs and $35 \%$ for SLO images has been achieved, when compared with manual counting. The colour fundus photograph method also showed superior reproducibility compared with the SLO technique.
\end{abstract}

Age-related macular degeneration (ARMD) is the commonest cause of registered blindness in Western society. ${ }^{1-4}$ Macular drusen are almost invariably present in patients with ARMD and their presence has been linked to development of sight-threatening maculopathy, such as retinal pigment epithelial detachment, retinal pigment epithelial atrophy and choroidal neovascularisation.

While drusen may not necessarily be accompanied by visual loss, they are prevalent in the elderly ${ }^{3-5}$ and features such as increasing number, size, degree of confluence and fluorescein angiographic behaviour are predictive of visual loss from exudative macular

From: ${ }^{1}$ Department of Ophthalmology and ${ }^{2}$ Department of Bio-Medical Physics, University of Aberdeen, Medical School, Foresterhill, Aberdeen AB9 2ZD, UK.

Correspondence to: Prof. J. V. Forrester, Dept of Ophthalmology, Medical School, Foresterhill, Aberdeen AB9 2ZD, UK. degeneration. ${ }^{6-11}$ Although neither prevention nor treatment of drusen has been established there is a need for accurate understanding of the natural history of drusen.

Most clinical studies in this area have relied on qualitative or semi-quantitative techniques by applying grading scales to fundus photographs or using photographic standards for comparison by observers. $^{6-9,12,13}$ Computer image analysis of fundus photographs has also been employed in this field. ${ }^{14,15}$ While human observers can count drusen numbers accurately if they are not too numerous, they have difficulty if large numbers of drusen are present. Furthermore, it may be argued that measurement of the area of the macula affected is more relevant than number of drusen as this may represent the extent of the dysfunction of the retinal pigment epithelium. The measurement of such areas lends itself to computerised image processing, as these techniques may be more accurate and less labour intensive than area estimation by human observers.

There have been reports of the appearance of macular drusen when imaged by the scanning laser ophthalmoscope (SLO) ${ }^{16,17}$ In particular, macular drusen have a characteristic appearance when imaged using the indirect mode of the instrument to detect scattered light returning from the fundus. In this mode of operation, the greater penetration of. infrared light, compared with visible wavelengths, permits identification of macular drusen in a characteristic 'moon surface' pattern with the edges of drusen clearly displayed. ${ }^{17}$

In this paper we describe the development of new methods for automated detection of drusen from colour fundus photographs and SLO images, using computer image analysis. The accuracy of these methods has been compared by performing manual 
Table I. Patient details

\begin{tabular}{ccl}
\hline \multicolumn{4}{c}{ Age (years) } \\
Patient & and sex & Drusen appearance \\
\hline A & $73 \mathrm{M}$ & $\begin{array}{l}\text { Scattered hard drusen + confluent soft } \\
\text { drusen at fovea }\end{array}$ \\
B & $73 \mathrm{M}$ & $\begin{array}{l}\text { Scattered hard drusen + confluent soft } \\
\text { drusen at fovea }\end{array}$ \\
C & $72 \mathrm{~F}$ & $\begin{array}{l}\text { Small hard drusen } \\
\text { Darge confluent soft drusen }\end{array}$ \\
$\mathrm{E}$ & $65 \mathrm{M}$ & $\begin{array}{l}\text { Large } \\
\text { Scattered soft drusen }\end{array}$ \\
$\mathrm{F}$ & $64 \mathrm{M}$ & Confluent soft drusen \\
\hline
\end{tabular}

counting of drusen. Estimation of the reproducibility of such methods is also reported.

\section{METHODS}

Five patients attending the outpatient department, Aberdeen Royal Infirmary, were selected for this study. Patients gave informed consent and this study was approved by the hospital ethics committee. Clinical details are shown in Table I.

\section{Image Capture}

All patients had fundus photography using a Topcon TRC 50 VT fundus camera set at $35^{\circ}$ field centred on the macula. Colour transparencies were then digitised by projection through a $550 \mathrm{~nm}$ interference filter using a highly collimated light source and focused onto an optically aligned Kodak MegaPlus charge-coupled device $(C C D)$ camera $(1320 \times 1024$ elements). Digital output from the camera was fed to the frame-grabber of a Series-151 image processing system (Imaging Technology, USA) permitting capture of each frame at a resolution of $1024 \times$ 1024 pixels. The system was controlled by a SUN IPX workstation (Sun Microsystems, USA) through the Visilog image processing software (Noesis, France).

SLO imaging of the same patients was carried out on the same day using a custom-built device. The construction and performance characteristics of this instrument have been detailed elsewhere. ${ }^{18,19}$ An infrared diode laser $(830 \mathrm{~nm})$ was used for illumination, at an incident power at the cornea of $200 \mu \mathrm{W} /$ $\mathrm{cm}^{2}$. The indirect mode was used to create images for processing by placing a central stop in front of the detector. This permitted the passage of only scattered light back to the detector and removed any directly reflected light. Suitable images were grabbed and stored on a PC. The SLO images were of a $25^{\circ}$ field and at a resolution of $740 \times 511$ pixels. These images were then transferred to the computer workstation for subsequent image analysis.

\section{Pre-processing}

Illumination Uniformity Correction. Digitised colour slides were pre-processed to correct for any variation in illumination caused by the projection process. ${ }^{20}$ The resultant image, free of artefactual variations in image intensity, was saved prior to further processing.

Image Registration. In order to compare accurately the images from fundus photography and SLO imaging it was necessary to register the images and select areas for drusen quantitation. After illumination uniformity correction the digitised colour slides were sampled by a factor of 2 to create $512 \times 512$ pixel images. SLO images were converted to $512 \times$ 512 images by cutting a strip 228 pixels wide from one side of the original and by adding a single row of zero-intensity pixels to the top of the image. It was then possible, using the manual registration facility of the Visilog software, to warp the colour slide images so that they coincided with the SLO images, by selection of at least 12 corresponding points on the images. The resultant image pair for each eye examined (SLO and colour slide image) formed the basis of all subsequent image processing and manual drusen estimation, and will henceforth be referred to as the 'original' images.

\section{Image Processing: Colour Slides (Fig. 1)}

Drusen appear as structures which are brighter than the background fundus (Fig. 1, top left). However, the background brightness level is not uniform in fundus photographs as a result of both natural variations in pigmentation and uneven illumination of the fundus by the fundus camera. Since the most straightforward technique of identifying drusen is to detect high-intensity areas by thresholding, it is necessary to render the background more uniform to avoid bright areas of background fundus being identified, in error, as drusen. This is achieved by a combination of subtraction and division methods of shade-correction. The former is achieved by subtracting an image approximating to the background fundus from the original, and the latter by the division of the original by the 'background' image. This heavily smoothed 'background' image was created by application of a large median filter. ${ }^{21}$ The result of these corrections is to produce a more even background intensity (Fig. 1, top right).

For the purposes of this study drusen detection was carried out within a $256 \times 256$ area of interest (AOI) which was selected interactively and centred on the fovea, prior to thresholding. It is also possible to select automatically a certain area of the image or, indeed, the whole image for processing, but interactive selection of the AOI is preferred to avoid obvious false positives such as the optic disc. We have found that the most accurate method of thresholding is by a combination of two methods: global and local thresholding. ${ }^{22}$ The former was performed by creating a binary image which consisted of those pixels whose intensities were a chosen number of 


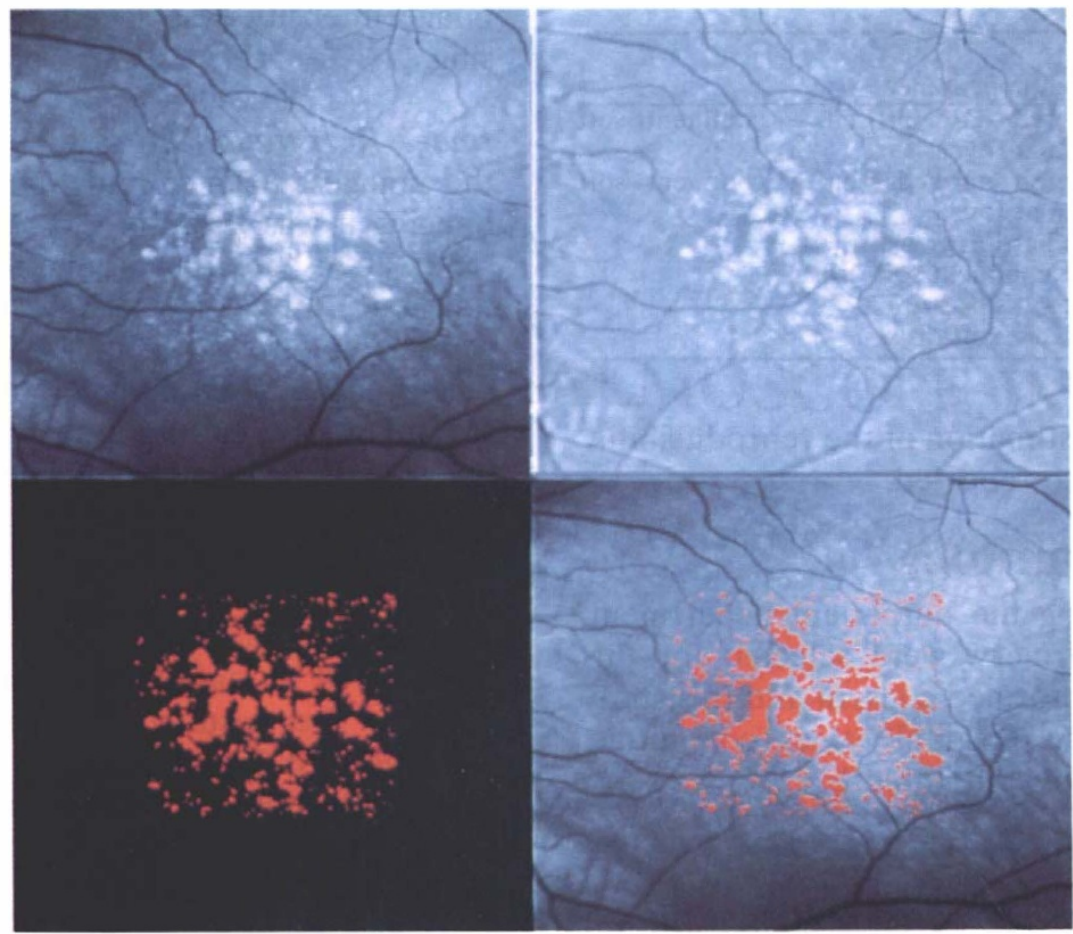

Fig. 1. Top left: digitised colour slide showing drusen as high-intensity structures compared with the background. Top right: Shade correction renders the background more uniform. Bottom left: Binary image after thresholding within the central $256 \times$ 256 pixel area of interest. Bottom right: Binary image overlaid onto original image to demonstrate detected drusen.

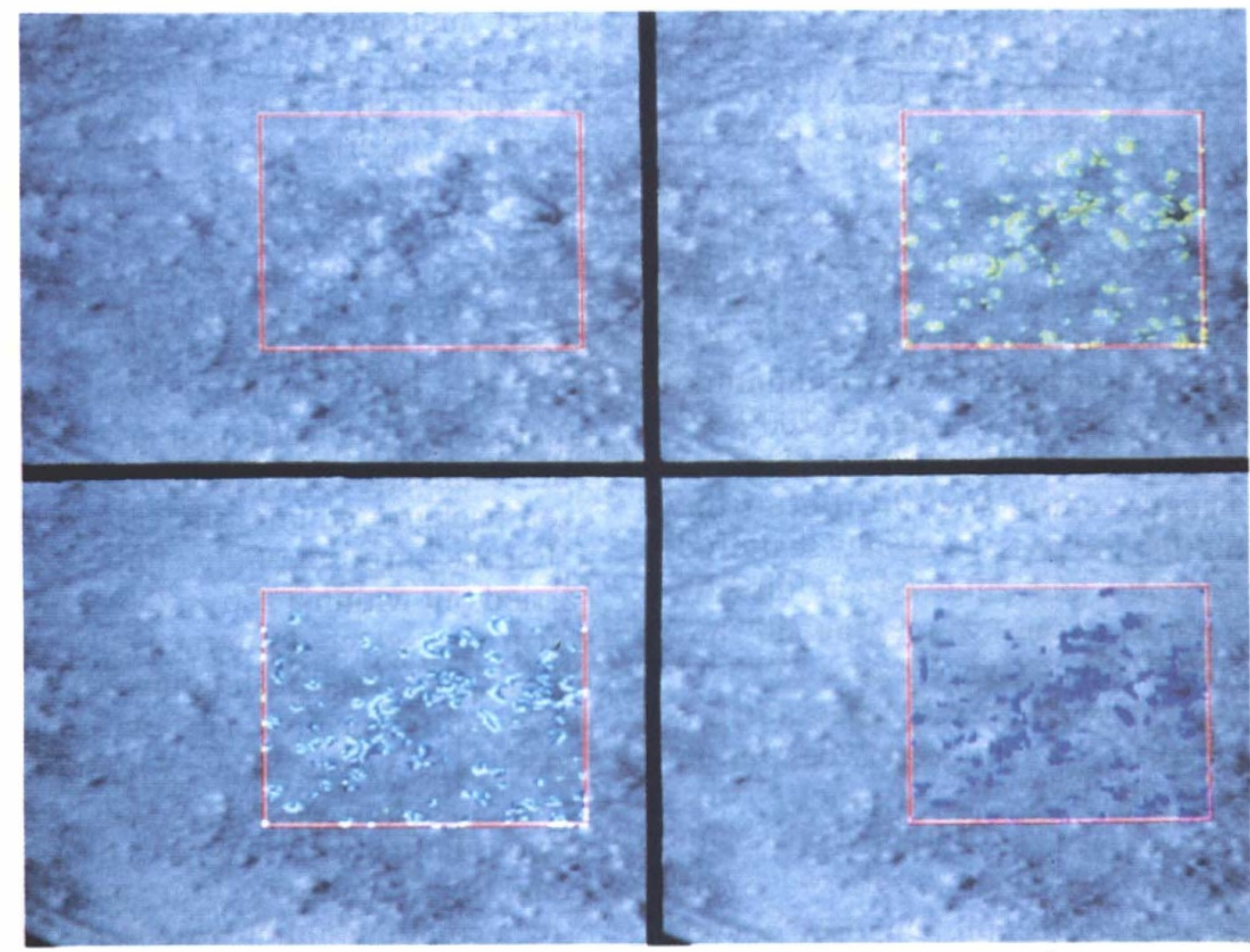

Fig. 2. Top left: SLO image of macular drusen with typical 'moon surface' appearance. The central macular area is delineated by the red square. Top right: Drusen edges have been detected using a gradient edge-detection filter. Bottom left: Using the detected edges as seeds for region-growing, the centres of drusen are filled. Bottom right: Detected drusen are shown overlying the original image. 
grey levels greater than the modal value of the shade-corrected image (typically 10-30 grey levels). Local thresholding was performed within the AOI by dividing the area into 64 separate areas of $32 \times 32$ pixels and applying a threshold to each area which was, again, a fixed number of grey levels above the modal value for that area. Typically, the threshold was 5 grey levels below the global threshold. This local thresholding technique permitted improved detection of faint drusen which were not identified by the global threshold. The binary images from the global and local thresholding techniques were combined by application of a logical 'or' operator. The total pixel area of the resultant binary image (Fig. 1, bottom left) could then be measured. Alternatively each binary object, i.e. single drusen, could be measured in terms of its area, perimeter, aspect ratio, shape and a number of other factors related to the underlying grey levels in the original image (Fig. 1, bottom right).

Seven separate threshold levels for drusen detection were applied to all six images and the optimum threshold level determined by comparison with drusen counted manually from the same images (see below).

\section{Image Processing: SLO Images (Fig. 2)}

Indirect mode infrared SLO images of fundi with macular drusen showed a 'moon surface' appearance, with drusen edges appearing as 'shadows' (Fig. 2, top left). However, the overall variation in image intensity of these images, when compared with digitised colour fundus photographs, is much lower. Our approach to drusen detection in these images was to employ a method to detect drusen edges and then to subsequently 'fill in' the central portion of the drusen using a 'region-growing' technique.

Edge detection was carried out by application of a gradient edge-detection kernel to the original SLO image. There were four separate steps to amplify pixels which formed the left, right, upper and lower borders of the drusen. From the resultant images a threshold level was selected automatically to create four binary images which corresponded to the drusen edges (Fig. 2, top right). These binary images then served as the starting point for a region-growing algorithm to fill the centres of detected drusen. Each binary object was taken in turn and, by using the grey scale information from the original SLO image, the domain of the drusen was filled in. For example, if one took a binary object which represented the left side of a particular drusen then, with the regiongrowing algorithm, the computer will add pixels to the right side of the binary object as long as the pixels in the underlying grey scale image do not exceed a certain parameter which, in this case, was the grey level gradient of the three immediate pixels to the right. This process was repeated for the right edges of drusen by region-growing to the left, for upper edges by region-growing downwards and for lower edges by region-growing upwards, thus filling the centre of each drusen (Fig. 2, bottom left). By carrying out this process from four sides of a potential drusen, the detection of drusen which may have one or two indistinct edges, can be improved (Fig. 2, bottom right).

Each image was processed at three different edgedetection thresholds and also using three different gradients for region-growing for comparison with manually counted drusen from the same images. The same AOI was used for these images as for the original colour slides.

\section{Manual Counting}

Using the $512 \times 512$ registered colour slide and SLO images a process of manual estimation of drusen area was carried out in order to create 'gold standards' for comparison with automated methods. This process was carried out prior to automated drusen counting to avoid observer bias. Since the computer software did not permit the interactive encirclement and filling in of observed drusen to create a binary image, an alternative method was used.

Original images were photographed from the screen of an Eizo high-resolution monitor $(1280 \times$ 1024 pixels) and developed into $10 \times 8$ inch prints. An acetate sheet was overlaid for marking of drusen with a permanent marker pen. Accurate alignment of acetate sheet and photographic print was maintained by use of a Kodak registration punch. The computer image was used to identify a drusen and its limits, and these were then marked on the acetate sheet. By frequent referral to the original computer image inaccuracies caused by the photographic processing were minimised. Any feature suspected of being part of a drusen was marked so that only areas which the observers felt were definitely background were left unshaded. The acetate sheet was then photographed and the resultant negative was digitised in the same fashion as the colour slides. This digitised image was converted to a binary image by thresholding and registered onto the original computer image prior to area measurement by the computer.

\section{Accuracy Assessment}

For original colour slide and SLO images, a comparison was made between the automated counting method at a particular threshold level and the 'gold standard' area from manual counting. By use of a logical 'and' operator on the automated and manual binary images, the overlap of detected pixels yielded the number of true positive pixels detected by the automated methods. The difference between the true positive pixels and the remainder of the 


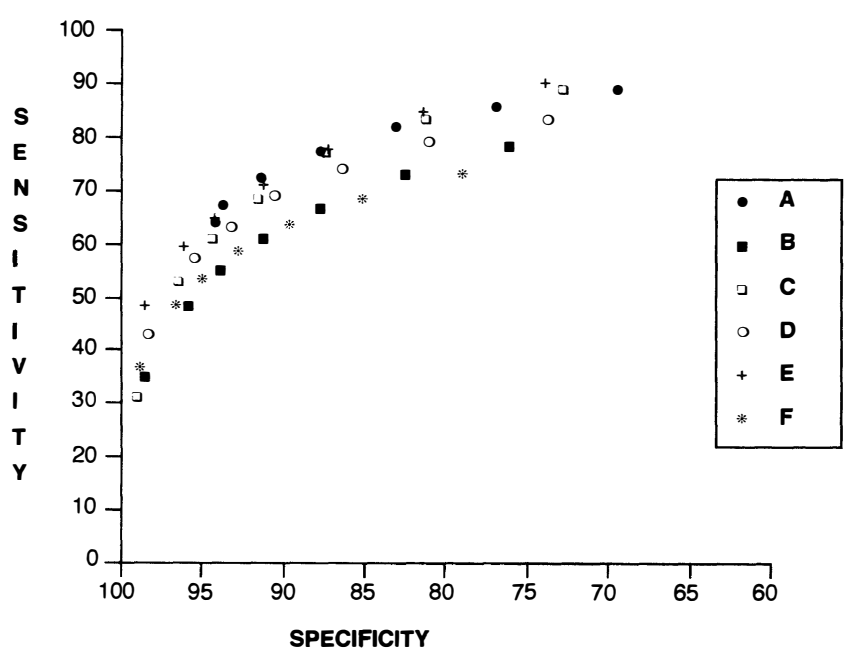

(a)

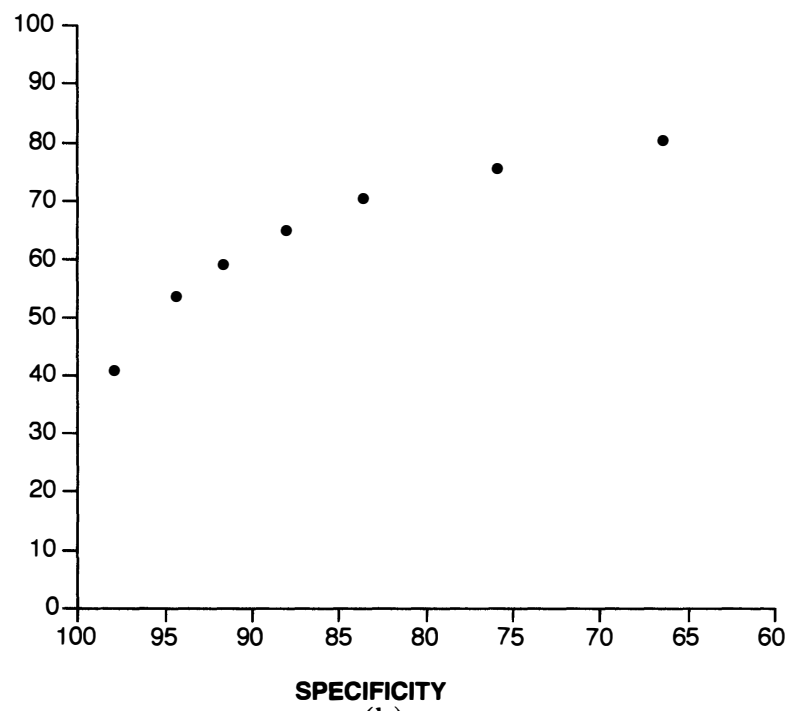

(b)

Fig. 3. (a) Individual receiver operating characteristic (ROC) curves for the colour slide drusen detection method. Each image is processed at a range of thresholds to create the curves. (b) Mean ROC curve for the colour slide method. Results from individual images at each threshold level have been summated.

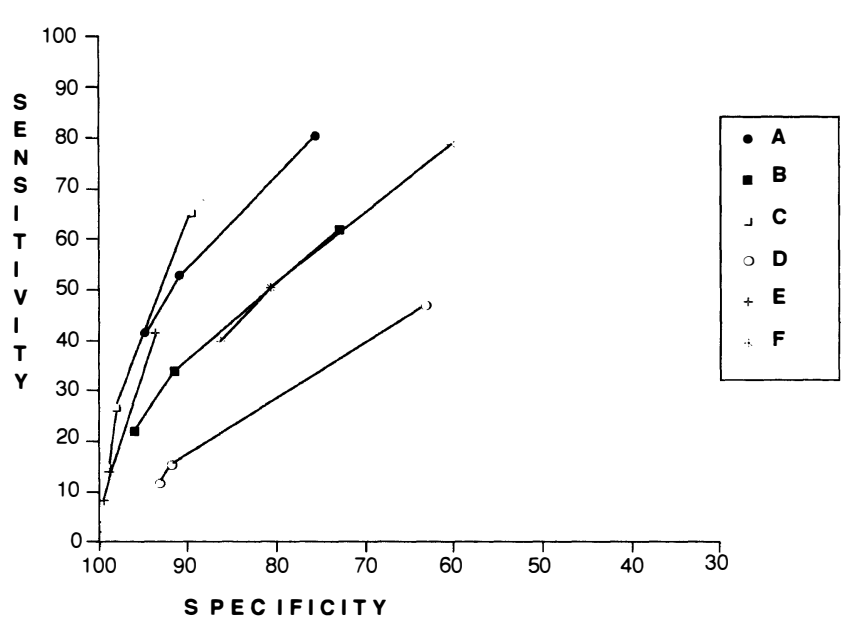

(a)

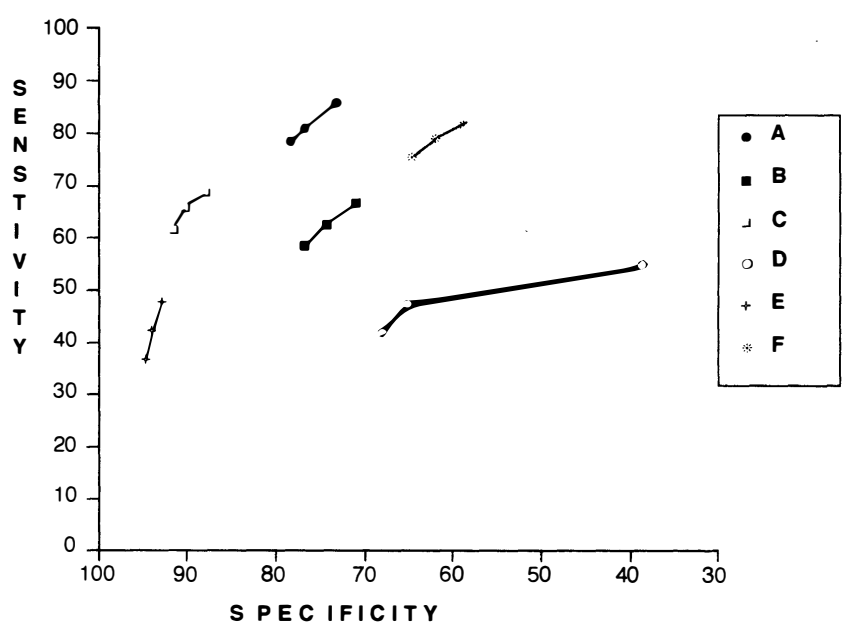

(b)

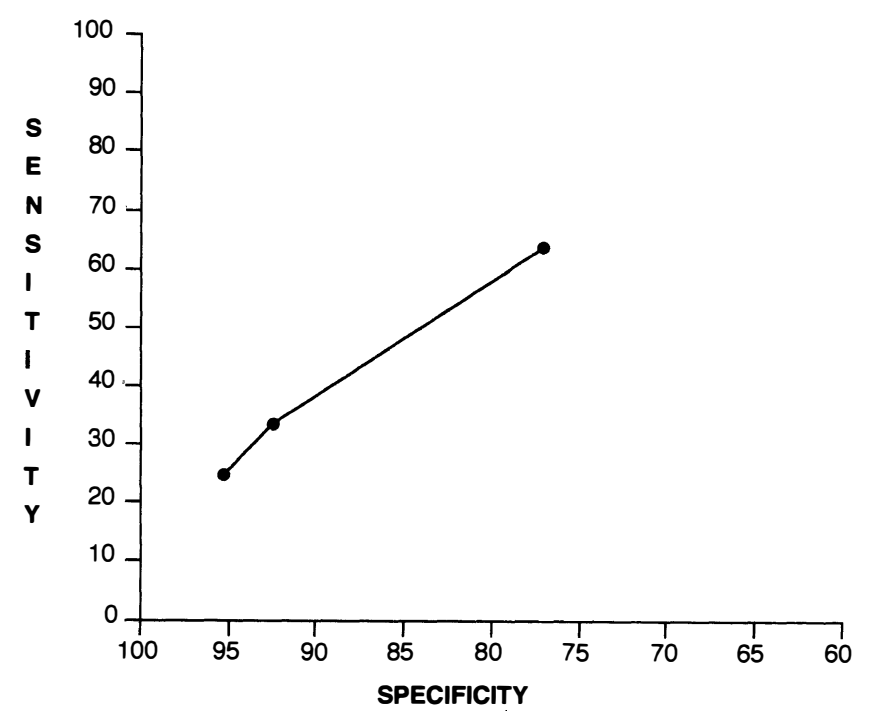

(c)

Fig. 4. (a) ROC curves for drusen detection from SLO images. Each image was processed at three edge-detection thresholds to create the curves. (b) ROC curves for drusen detection from SLO images. Edge detection was maintained at the same threshold while the parameters for region growing were varied for each image. (c) Mean ROC curve for SLO images created from summation of data at each threshold in $(a)$. 
Table II. Gold standard area counts

\begin{tabular}{ccc}
\hline & \multicolumn{2}{c}{ Pixel area } \\
\cline { 2 - 3 } Patient code & Colour slide & SLO image \\
\hline A & 9261 & 7640 \\
B & 19497 & 23508 \\
C & 512 & 2630 \\
D & 31628 & 30779 \\
E & 10069 & 18290 \\
F & 32270 & 25026 \\
Total & 103237 & 107873 \\
\hline
\end{tabular}

automated binary image constituted the false positive pixel count, and by subtraction of the manual binary pixel count from the size of the total area of the area of interest (65 536 pixels) the true negative pixel area was calculated. From these figures the sensitivity of a given test was calculated as:

$$
\frac{\text { Detected true positive pixels }}{\text { Total true positive pixels }} \times 100(\%)
$$

and specificity of the test as:

True negative pixels - false positive pixels

True negative pixels 100

$\times 100(\%)$

Sensitivity and specificity values were calculated for each threshold level that had been applied to each SLO and colour slide image.

\section{Reproducibility}

By taking a second image from a patient at the same time it is possible to assess reproducibility of the techniques used. Second colour slides of drusen from two eyes underwent the same pre-processing of illumination correction and registration as the originals. Using the same AOIs on each, the automated detection program was applied at the same threshold and the areas detected were compared. The threshold selected was the level which achieved just greater than $90 \%$ specificity.

For SLO images, second images from two eyes were registered to the originals and processed in the same fashion, using the lowest threshold for edge detection and the intermediate gradient for regiongrowing to permit comparison of detected areas.

\section{RESULTS}

The sensitivity achieved by the use of a particular threshold level applied to each image can be plotted against specificity in the form of a receiver operating characteristic (ROC) curve.

For the six colour slide images a range of seven threshold levels was applied to each in turn and the results were then plotted as the ROC curves shown in Fig. 3a. The spread of these data is small. Since the same range of thresholds was applied to all images, these results can be summated and a mean ROC curve for automated drusen detection is shown in
Table III. Reproducibility data

\begin{tabular}{lccc}
\hline & $\begin{array}{c}\text { First image } \\
\text { drusen area }\end{array}$ & $\begin{array}{c}\text { Second image } \\
\text { drusen area }\end{array}$ & $\begin{array}{c}\text { Area difference } \\
\text { (\% of mean })\end{array}$ \\
\hline Colour slides & & & \\
Patient 1 & 14038 & 13960 & 0.56 \\
Patient 2 & 16658 & 16487 & 1.03 \\
SLO images & & & \\
Patient X & 17449 & 18006 & 3.14 \\
Patient Y & 10565 & 12093 & 13.48 \\
\hline
\end{tabular}

Fig. 3b. As the threshold is lowered the sensitivity rises, but at the expense of specificity. At a specificity of $90 \%$ the mean sensitivity is greater than $60 \%$.

Fig. 4a shows the ROC curves plotted in a similar manner for the six SLO images, when three different edge-detection thresholds were applied to detect drusen. These data show greater variation between images and the overall sensitivity tends to be low. Fig. $4 \mathrm{~b}$ shows the ROC curves created by maintaining the same edge-detection threshold but varying the level of region-growing. From these curves it can be seen that altering this parameter does not in general result in great changes in sensitivity or specificity. The mean ROC curve for edge-detection threshold variation for SLO images is shown in Fig. 4c. Here mean sensitivity is only $35 \%$ at a specificity of $90 \%$, although some individual images (patients $\mathrm{A}$ and C) achieve a sensitivity of $50 \%$ for $90 \%$ specificity.

Since colour slide and SLO images were spatially registered it was possible to compare the 'gold standard' areas obtained by manual counting of the two imaging modalities (Table II). There is some variation between data pairs but no statistically significant difference $(p>0.05$ using Student's $t$-test for paired data).

Reproducibility results from colour slide and SLO images are presented in Table III. The variation, which is expressed as the percentage of mean difference, is seen to be much lower for the colour slide method.

\section{DISCUSSION}

Accurate quantification of pathological features in the fundus, such as macular drusen, permits better understanding of the natural history of the condition. Furthermore, the effect of any putative therapeutic agent can be more rapidly assessed than if qualitative methods are employed. In patients with macular drusen the factors associated with increased risk of visual loss, such as number, size, angiographic features and confluence, have been identified in qualitative and semi-quantitative studies. ${ }^{6-11} \mathrm{Com}$ puter image analysis techniques may allow further evaluation of these factors as well as performing these tasks with high repeatability in a fraction of the time required by a human observer. Computer image analysis techniques are employed with increasing frequency in the quantification of conditions such as 
optic disc pathology, diabetic retinopathy and conditions associated with fluorescein angiographic leakage. ${ }^{21-25}$

Previous work on the computer image analysis of macular drusen has been reported but using different techniques from those employed here. ${ }^{14,15}$ This earlier method relied on the subdivision of the image into small $8 \times 8$ pixel areas and the automatic application of an algorithm to determine whether each area contained parts of individual drusen. From this, threshold levels were selected and applied to the whole image. While this method avoided the use of arbitrarily selected thresholds, there was, unfortunately, a high false positive rate due, firstly, to the erroneous detection of areas close to retinal vessels and, secondly, to the use of unsuitable threshold values for large patches of background not containing drusen. This necessitated considerable operator intervention to eliminate false positives in each image studied. The accuracy of this technique was not measured by comparison with manual counting and so comparison with the present technique is not possible. The reproducibility for the earlier technique was acceptable after manual editing (coefficient of variation $<4 \%$ ).

From the data presented here there is no significant difference in the areas of the fundus containing drusen when fundus photographs and infrared SLO images of the same patient are compared by manual counting (see Table II). It is unlikely that a significant difference would be apparent from a data set containing only six pairs of images and, indeed, there does not appear to be a trend, with half the image pairs showing a greater area on the registered colour slide while the remainder show a greater area on the SLO image.

The accuracy of the techniques proposed in this paper is less than ideal. While some thresholds for either imaging technique yield high specificity, the sensitivity is rather low. At a level of $90 \%$ specificity there is a sensitivity of $60 \%$ for the colour slide method and of only $35 \%$ for the SLO image method. There are a number of reasons for this low sensitivity, which are related to the images and the processing employed.

Firstly, it can be argued that the gold standards may have contained inaccuracies because of the method employed. Since only areas which the observers felt were definitely background were left unshaded it is possible that the measurement of true positive area was falsely high. It is quite possible that there could be some variation in the opinion of different observers concerning areas where drusen appear faint. The method for creation of gold standards was selected as it was felt that it could be applied consistently to all images.

Secondly, the automated technique for drusen detection in colour slides is limited to a certain extent by the method of background shade-correction. A spatially fine shade-correction algorithm can be used but, while this creates an even background over which one may apply a certain threshold, larger structures such as soft drusen become shadecorrected and so disappear from the image. Thus a coarser shade-correction was employed, which left some non-uniformity in the background shading. With this technique it was not possible to apply a low threshold to the whole image because of the resultant high false positive rate. While most drusen were identified by the computer technique after application of such a global threshold, there was often a thin rim of drusen edge not detected, which resulted in a reduction of the area measured. In an attempt to improve the detection of faint drusen and the edges of obvious drusen, a local thresholding technique was applied to small $32 \times 32$ pixel blocks using a threshold level set to a value below the global threshold. This limited the detection of false positives.

Thirdly, the reduction in resolution of digitised colour slides from $1024 \times 1024$ pixels to $512 \times 512$ pixels to allow for image registration with the SLO images may have reduced the accuracy of the results slightly. To this end, a revised method for the higherresolution images is in preparation.

False positive results from such thresholding techniques originate from two possible sources. Firstly, the use of low thresholds causes large areas of background to be erroneously identified as drusen and, secondly, there may be other pathology present such as retinal exudates, chorioretinal atrophy or myelinated fibres, which is detected due to high reflectance. It is thus necessary to apply such techniques as described here to images in which the diagnosis has been confirmed by an ophthalmologist and not for screening for a specific disease.

In the SLO imaging technique macular drusen are visualised as they scatter infrared laser light. When imaging the fundus with infrared light in the direct mode, drusen are almost invisible as they do not directly reflect this wavelength. The indirect mode images are very uniform but the drusen edges are visualised as light or dark borders. The application of a simple thresholding technique was thus inappropriate and an edge-detection method was employed. The low sensitivity of this technique stems directly from the variability in the observed edges. Small hard drusen tend to have sharp edges which are relatively easily detected, but some soft larger drusen have faint edges which were difficult to detect. Since the image processing technique relied on the use of detected edges as a seed for region-growing, then the sensitivity for detection was variable and depended to some extent on the nature of the drusen edges in 
any given image. Lowering the threshold for edge detection to improve sensitivity, results in an unacceptable specificity. Thus, despite the characteristic appearance of drusen in this mode of imaging, these images pose a difficult problem for image processing techniques. Notwithstanding these problems, the practical advantages of the SLO compared with fundus photography, in terms of the avoidance of photographic processing, low light levels required and immediate access to directly digitised images for image processing, mean that this instrument may still have a role in imaging of drusen. Despite the fact that the penetrance of infrared light into the fundus is greater than for visible wavelengths drusen show a high reflectance compared with background when imaged with visible wavelengths. Thus it may be more appropriate to develop programs for drusen detection using visible wavelengths with the SLO.

Given existing techniques, drusen area estimation from indirect mode infrared SLO images or colour fundus photographs is an impractical proposition because of low sensitivity which is principally due to difficulties in performing satisfactory image processing on such relatively low-contrast images. Recent experience of confocal mode SLO imaging with visible wavelengths in our department has shown considerably superior image contrast compared with fundus photographs. These images may be more suitable for processing and this is the subject of continuing research.

The authors are grateful for photographic assistance provided by Mr R. Hutcheon and Mrs A. Farrow. This work was supported by funds from an ACTR Fellowship from the Scottish Home and Health Department, the Wellcome Trust and Scotia Pharmaceuticals Ltd.

Key words: Fundus photography, Image processing - computer assisted, Macular degeneration, Ophthalmoscopy - scanning laser, Retinal drusen.

\section{REFERENCES}

1. Grey RHB, Burns-Cox CJ, Hughes A. Blind and partially sighted registration in Avon. Br J Ophthalmol 1989;73:988-94.

2. Thompson JR, Du L, Rosenthal AR. Recent trends in the registration of blindness and partial sight in Leicester. Br J Ophthalmol 1989;73:95-9.

3. Klein R, Klein BEK, Linton KLP. Prevalence of age related maculopathy: the Beaver Dam Eye Study. Ophthalmology 1992;99:933-43.

4. Leibowitz HM, Krueger DE, Maunder LR, et al. The Framingham Eye Study monograph. Surv Ophthalmol 1980;24(Suppl):335-610.

5. Coffey AJH, Brownstein S. The prevalence of macular drusen in postmortem eyes. Am J Ophthalmol 1986; 102:164-71.

6. Strahlman ER, Fine SL, Hillis A. The second eye of patients with senile macular degeneration. Arch Ophthalmol 1983;101:1191-3.

7. Gregor Z, Bird AC, Chisholm IH. Senile disciform macular degeneration in the second eye. $\mathrm{Br} \mathrm{J}$ Ophthalmol 1977;61:141-7.

8. Gass JDM. Drusen and disciform macular detachment and degeneration. Arch Ophthalmol 1973;90:206-17.

9. Smiddy WE, Fine SL. Prognosis of patients with bilateral macular drusen. Ophthalmology 1984; 91:271-7.

10. Chuang EL, Bird AC. The pathogenesis of tears of the retinal pigment epithelium. Am J Ophthalmol 1988;105:285-90.

11. Pauleikhoff D, Barondes MJ, Minassian D, Chisholm IH, Bird AC. Drusen as risk factors in age related macular disease. Am J Ophthalmol 1990;109:38-43.

12. Bressler NM, Bressler SB, Fine SL, Taylor HR. The grading and prevalence of macular degeneration in Chesapeake Bay watermen. Arch Ophthalmol 1989; 107:847-52.

13. Klein R, Davis MD, Magli YL, Segal P, Klein BEK, Hubbard L. The Wisconsin age related maculopathy grading system. Ophthalmology 1991;98:1128-34.

14. Peli E, Lahav M. Drusen measurement from fundus photographs using computer image analysis. Ophthalmology 1986;93:1575-80.

15. Sebag M, Peli E, Lahav M. Image analysis of changes in drusen area. Acta Ophthalmol (Copenh) 1991; 69:603-10.

16. Van de Velde FJ, Jalkh AE, Katsumi O, Hirose T, Timberlake GT, Schepens CL. Clinical scanning laser ophthalmoscope applications: an overview. In: Nasemann JE, Burk ROW, eds. Scanning laser ophthalmoscopy and tomography. Munich: Quintessenz Verlag, 1990:35-48.

17. Manivannan A, Kirkpatrick JNP, Sharp PF, Forrester JV. Clinical investigation of an infrared digital scanning laser ophthalmoscope. Br J Ophthalmol 1994;78:84-90.

18. Manivannan A, Sharp PF, Phillips RP, Forrester JV. Digital fundus imaging using a scanning laser ophthalmoscope. Physiol Meas 1993;14:43-56

19. Manivannan A, Sharp PF, Forrester JV. Performance measurements of an infrared digital scanning laser ophthalmoscope. Physiol Meas 1994;15:317-24.

20. Spencer T. Digital imaging of the retina. $\mathrm{PhD}$ thesis, University of Aberdeen, 1992.

21. Spencer T, Phillips RP, Sharp PF, Forrester JV. Automated detection and quantification of microaneurysms in fluorescein angiograms. Graefes Arch Clin Exp Ophthalmol 1992;230:36-41.

22. Phillips RP, Forrester JV, Sharp PF. Automated detection and quantification of retinal exudates. Graefes Arch Clin Exp Ophthalmol 1993;231:90-4.

23. Chihara E, Takahashi F, Chihara K. Assessment of optic disc topography with the scanning laser ophthalmoscope. Graefes Arch Clin Exp Ophthalmol 1993; 231:1-6.

24. Kruse FE, Burk ROW, Volcker HE, Zinser G, Harbarth U. Reproducibility of topographic measurement of the optic nerve head with laser tomographic scanning. Ophthalmology 1989;96:1320-4.

25. Phillips RP, Ross PG, Tyska M, Sharp PF, Forrester JV. Detection and quantification of hyperfluorescent leakage by computer analysis of fundus fluorescein angiograms. Graefes Arch Clin Exp Ophthalmol 1991;229:329-35. 